\title{
Cluster based Sleep/Wakeup Scheduling Technique for WSN
}

\author{
Sasikala V \\ Professor, DRBCCC Hindu College, \\ Chennai, Tamil Nadu
}

\author{
C. Chandrasekar,Ph.D \\ Associate Professor, Dept. of Computer Science, \\ Periyar University, \\ Salem - 636 011, Tamil Nadu, India
}

\begin{abstract}
Wireless sensor network (WSN) is an emerging technology in the today's world. The WSN has been a prominent technology in many fields so it is very important to decrease the energy consumption by the network and increase the network's life. To achieve this, cluster based Sleep/Wake scheduling technique is proposed. In this approach, some nodes are considered as initiator nodes through which the cluster heads are decided. In each cluster, the cluster head selects a node with the highest energy and keep it in active mode and sends the remaining nodes into the sleep mode. Then again the initiator nodes collect the residual energies details of these clusters and compare with the standard threshold value. If the cluster energy is less than the threshold value then that particular cluster will be sent into the sleep mode. At the last, the transmission will be done by the active clusters to the sink node. Through this way it is possible to decrease the energy consumption of the network and increase the network's life time. By simulation results, it is proved that the proposed technique reduces the energy consumption and increases the packet delivery ratio.
\end{abstract}

\section{Keywords}

Wireless Sensor Network (WSN), Sleep/Wake Scheduling, Cluster Head (CH), Initiator Nodes (I).

\section{INTRODUCTION}

Generally a Wireless sensor network (WSN) is a collection of sensor nodes interconnected by wireless communication channels. Where each sensor node is a small device that can collect data from its surrounding area, carry out simple computations, and communicate with other sensors or with the base station (BS). Because of this property the wireless sensor network has become a very important topic with the rapid development that is vulnerable to a wide range of attacks due to deployment in the hostile environment. A WSN is a large network of resource-constrained sensor nodes with multiple preset functions, such as sensing and processing with number of low-cost, resource limited sensor nodes to sense important data related to environment and to transmit it to sink node that provides gateway functionality to another network, or an access point for human interface. These sensor networks are composed of energy constrained nodes embedding limited transmission, processing and sensing capabilities. Therefore network lifecycle becomes short and hence energy-efficient technique implementation becomes an important requirement for WSN. $[1][2][3][12]$

\subsection{Needs for Power Efficient Scheduling}

In order to provide high-quality data service, a multilevel of sensing coverage and network connectivity is needed in the practical implementation of a WSN. In wireless networks with battery-operated devices, energy saving mechanisms is of paramount importance in order to maximize network lifetime. Energy conservation is important during periods with no activity and also during occurrence of events. It is critical to reduce traffic overhearing since the transceiver consumes similar energy for idle listening as transmission. In WSNs, a sleep-wake duty cycling has been adopted for energy efficiency and conservation, since each sensor node is typically equipped with a battery which is power-limited.

In wireless networks cluster is a group of nodes which is generally considered to be a scalable method to manage large sensor networks and each cluster consists of a single cluster head $(\mathrm{CH})$.

The network sensors nodes can be managed locally by a cluster head in a cluster - a node elected to coordinate the nodes within the cluster and to be responsible for communication between the cluster and the base station or other cluster heads. Clusters provide a convenient framework for resource management, data fusion, and local decision making. Since in a cluster all the nodes will be wake state to communicate with the cluster head. When this communication takes place without considering the energy of the nodes in the cluster. During this process all the nodes in the cluster consumes energy no matter what the nodes are capable of transmitting data.

So, in order to reduce this consuming energy by the nodes which are not capable of transmitting data or to communicate with in the cluster to the cluster head a sleep/wake scheduling technique is implemented in the network. Through this sleep/wake scheduling process, it is possible to conserve energy in the network. This is possible by dividing the node of a cluster into active nodes and the inactive nodes. The active nodes are the nodes which are with the high energy and which are capable of communicate with the cluster head and the inactive nodes are the nodes which are with the low energy and these nodes are not capable of communicating with the cluster head. These inactive nodes with the low energy are sent to the sleep mode. And the remaining nodes are kept in the wake mode which is capable of communication to the cluster head. Through this way it is possible to conserve the network energy and also it is possible to increase the network's life.

\section{$[4][5][6][7][8][9][14]$}

\subsection{Advantages of Scheduling}

- With the sleep/wake scheduling the sensors within the same cluster are densely deployed, the connectivity of the area covered by active sensors can be guaranteed. [4]

- If a source has no more traffic to send, the slot assignments along the flow path from the source to the sink will timeout. Thus nodes can adjust their sleep schedules according to the change of traffic patterns, which is important for energy conservation. [6][15]

- $\quad$ For continuous monitoring systems, synchronization-based sleep/wake scheduling schemes are often used because the traffic pattern is periodic. [9] 
- Energy consumption can be controlled when the sleep/wake scheduling is applied to the node of the network i.e. when a task has to be done only some of the nodes are assigned through a work message. [4][10]

\subsection{Issues of scheduling}

- If two neighboring clusters are not densely connected, they cannot be merged into a larger one [4].

- A cluster which broadcasts a merging request would not accept a merging request from other clusters so the cluster should send a message to the other cluster otherwise it may lead to the time consumption [4].

- Ideally, in a sensor network, a scheduling protocol must determine a transmission schedule for each packet otherwise collisions may take place [6][15].

- Time consumption can be more since the transmitted data packets visits all nodes which comes in the way and it will wait till the reception process is completed [7].

- When the sensor nodes are divided into multiple clusters only a few sensors are selected from different clusters to be in active status, if this repeats and the same nodes are kept inactive maximum times, then it may cause problem to the network in the future.

\subsection{Problem Identification}

In [16], a technique which combines energy efficiency and multiple path selection for data fusion in WSN was proposed. The network is partitioned into various clusters and the node with highest residual energy is selected as the cluster head. The sink computes multiple paths to each cluster head for data transmission. The distributed source coding and the lifting scheme wavelet transform are used for compressing the data at the $\mathrm{CH}$. During each round of transmission, the path is changed in a round robin manner, to conserve the energy. This process is repeated for each cluster.

But this approach consumes more energy since all the nodes in the network stay in active mode without transmitting anything. This leads to the decreasing of network's lifetime. To overcome this, this paper proposes a guaranteed distributed sleep/wake scheduling scheme, where only the transmitting node stay in active mode and the other nodes goes to the sleep mode.

\section{LITERATURE REVIEW}

Guofang Nan et al., [4] have proposed a coverage-guaranteed distributed sleep/wake scheduling scheme. The main purpose of this approach is to prolonging the network lifetime while guaranteeing network coverage. Here in this approach the sensor nodes are divided into clusters based on sensing coverage metrics and allows more than one node in each cluster to keep active simultaneously via a dynamic node selection mechanism. The main advantage of this approach is that it guarantees an efficient way of energy conservation in the network.

Sha Liu et al., [6] have contributed in an energy efficient sleep scheduling protocol called BSMac for sensor networks while maintaining high throughput and low latency which is based on a new architecture called BoostNet in which the base station broadcasts critical scheduling coordination information using large transmission range to reach all sensor nodes in one hop. The main contribution of this paper in energy conserving scheduling approach that conserves energy during event occurrence and does not require any transmissions by the sensors during periods of inactivity and they have used in-band high transmission power from the base station for network parameters optimization without requiring the second transceiver on sensor nodes.

Yan Wu et al., [7] have proposed an optimal sleep/wake scheduling algorithm, which satisfies a given message capture probability threshold with minimum energy consumption. In this approach is there is an inherent trade-off between energy consumption and message delivery performance. The advantage of this paper is that the authors have formulated an optimization problem which aims to set the capture probability threshold at each hop such that the network lifetime is maximized.

Bo Jiang et al., [10] have proposed an energy-aware, sleep scheduling algorithm called SSMTT to support multiple target tracking sensor networks. SSMTT leverages the awakening result of interfering targets to save the energy consumption on proactive wake-up communication. The authors have presented a solution that involves scheduling the sensor nodes' sleep pattern. Age of this approach is that it is possible to achieve better energy efficiency than handling multiple targets separately through single target tracking algorithms.

Chih-Min Chao et al., [11] have proposed quorum-based MAC protocol that enables sensor nodes to sleep longer under light loads. Since traffic flows toward the sink node in wireless sensor networks, a new concept, next hop group, is also proposed to reduce transmission latency. The advantage of this proposed approach is that the energy waste is reduced since nodes keep awake only at a specified time. And also this approach overcomes when a technique fails to adjust a sensor node's sleep duration based on its traffic load, thus causing either lower power efficiency or higher latency. Also, sensors may be deployed in hostile environments and may thus fail unexpectedly.

\section{PROBLEM IDENTIFICATION AND THE PROPOSED SOLUTION}

\subsection{Overview}

In this paper, the coverage-guaranteed distributed sleep/wake scheduling is technique is proposed. Here the cluster formation is formed from the previous work i.e. when an initiator node selects the energy information of the node and selects the cluster heads which has the highest residual energy. Through guaranteed distributed sleep/wake scheduling scheme, entire cluster's energy is compared which gives a connection value for each cluster in order to be in connect with the active nodes of other clusters. Once the selected cluster head node receives the joint request $\mathrm{J}_{\mathrm{REQ}}$ message from member nodes, it sends a joint reply message $\mathbf{J}_{\text {REP }}$ back to the nodes. Then if the particular cluster head's residual energy is greater than the given threshold value, only one node with higher energy will be in the active mode and the other nodes will be in the sleep mode and also the clusters with lower residual energy will also be goes to sleep mode. Then the $\mathrm{CH}$ transmits data to the sink node.

The advantage of the proposed extension is based on the cluster head's residual energy the cluster nodes are decided to be on active mode or in the sleep mode which increases the lifetime of the network.

\subsection{Cluster Head Selection}

In the cluster head selection, initially the node with the highest residual energy is selected as the cluster head to prolong the network lifetime. In order to the collect the network's information of the nearest sensor nodes, initiator nodes are considered. 
Assume that the sink node here has the knowledge about all the sensors nodes and their location in the network. The cluster head $(\mathrm{CH})$ is determined based upon the residual energy of the nodes which is found out by the initiator nodes (I). In a network, $\mathrm{CH}$ uses more energy than other nodes. But the network performance degrades when the energy of the cluster head goes down. To overcome this situation, the network energy consumption is to be balanced by ensuring that the $\mathrm{CH}$ keeps changing in a cluster depending upon its residual energy.

The process of the cluster head selection in the network takes place through the following steps.

- The initiator node I broadcasts a request message for energy $\left(\mathrm{E}_{\mathrm{REQ}}\right)$ with its own residual energy level $\left(\mathrm{RL}_{\text {ini }}\right)$ information to its surrounding nodes.

- The sensor node $S_{\mathrm{i}}$ compares its own energy level $\left(\mathrm{RL}_{\mathrm{i}}\right)$ with the initiator.

- If $\mathrm{RL}_{\mathrm{i}}>\mathrm{RL}_{\mathrm{ini}}$, then, $\mathrm{Si}$ sends a reply message for energy $\left(\mathrm{E}_{\mathrm{REP}}\right)$.

\section{Else}

$\mathrm{S}_{\mathrm{i}}$ waits for cluster head advertisement messages $\left(\mathrm{CH}_{\mathrm{ADV}}\right)$.

- The initiator node (I) selects the cluster head with maximum residual energy and the next initiator node is the node having the second maximum residual energy.

- The initiator node is changed every time when the energy level of the node decreases.

- After $\mathrm{CH}$ is selected by the initiator node (I), clusters are formed in the network.

- The nodes in the cluster broadcasts a $\mathrm{CH}_{\mathrm{ADV}}$ to the $\mathrm{CH}$ and $\mathrm{CH}$ sends it to the sink node along with the cluster ID.

- A join request message $\mathrm{J}_{\mathrm{REQ}}$ is transmitted by the member node along with $\mathrm{CH}_{\mathrm{ADV}}$.

The transmission range gets minimized since the initiators collect the energy information about the nearest sensors. The nodes having energy greater than the energy level of the initiator ensure minimization of $\mathrm{E}_{\mathrm{REP}}$ message transmission.

Once the selected cluster head node receives the $J_{R E Q}$ message from member nodes, it sends a joint reply message $J_{\text {REP }}$ back to the nodes. Then the $\mathrm{CH}$ transmits data to the sink node.

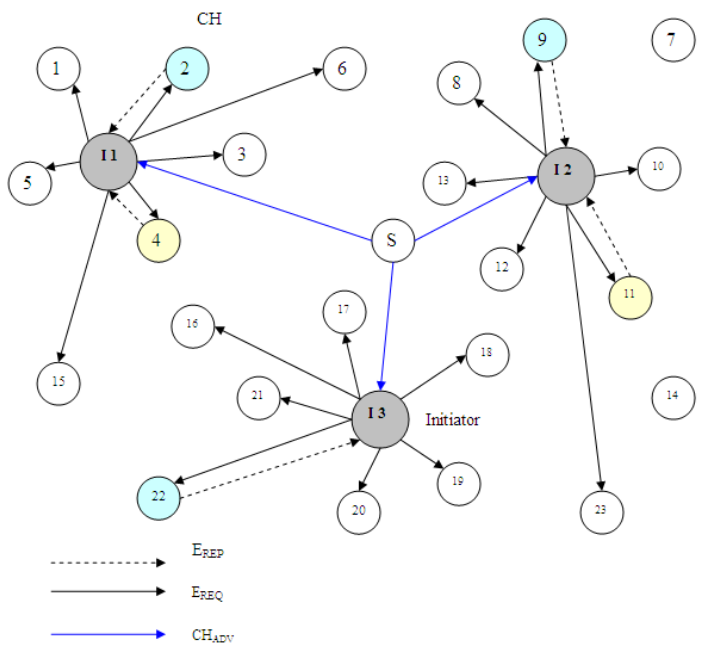

Figure 1: Selection of Cluster Head
In figure 1 , the Initiator I1, I2, and I3 sends energy request $\mathrm{E}_{\mathrm{REQ}}$ to all the surrounding nodes. For example, in this figure, when the node $\mathrm{I} 1$ sends $\mathrm{E}_{\mathrm{REQ}}$ to the nodes, the energy consumption of the nodes is compared with I1. Since node 2 has a higher energy level than I1 and so node 2 is selected as the cluster head and node 4 which has the next highest energy level is selected as the next initiator for cluster head selection.

Similarly, when the node $\mathrm{I} 2$ sends $\mathrm{E}_{\mathrm{REQ}}$ to the nodes, the energy consumption of the nodes is compared with I2. Here, node 9 has the highest energy level and it is selected as the Cluster head and the node 11 which has the next highest energy level is selected as the next initiator for cluster head selection.

Then when the node $\mathrm{I} 3$ sends $\mathrm{E}_{\mathrm{REQ}}$ to the surrounding nodes, the energy consumption of the nodes is compared with I3. Since node 22 has the highest energy level, it is selected as the cluster head and no other surrounding node has the next highest energy level other than initiator I3. So, node I3 is selected as the next initiator node.

\subsection{Sleep/Wake Scheduling}

Sleep/Wake scheduling is an every effective process where the network's energy is saved to a maximum extent. In the network once the cluster formation is done then the every cluster starts applying the process of sleep/wake scheduling. In order to save energy, only one or two nodes with highest residual energy in each cluster are required to keep active, while others will be kept on in the sleep mode.

At the beginning of this scheduling all the nodes in the cluster will be active in order to analyze the residual energies. This analysis is done to select an active node with the highest residual energy in a cluster. And this active node will undertake the sensing task in a cluster. The node which will undertake the sensing task will be decided by the cluster head. The cluster head sends a WORK message to order the selected node to perform its duty as an active node, moreover, one of which is told to be the head node in the next period. And also the cluster head sends a SLEEP message to all of the rest nodes. When the particular task is completed all the nodes in the sleep mode will send a WORK REQ to the cluster head to participate in node(s) selection when the next round comes. While receiving WORK REQs from all the sleeping nodes in the cluster, the head will run the process of selecting active nodes.

Here an initiator node sends the energy request $\left(\mathrm{E}_{\mathrm{REQ}}\right)$ to all the clusters and the clusters will sends an energy reply $\left(\mathrm{E}_{\mathrm{REP}}\right)$ to the initiator node. Through this process the initiator node will collect the residual energy details of all the clusters in the network. The energy of the each cluster will be compared with a standard threshold value which is fixed. If the initiator node finds any cluster having low residual energy than the fixed standard threshold value than that particular cluster will be sent into the sleep mode till the particular transmission is completed in the network. Through this way the network's energy will be consumed in a limited manner. 


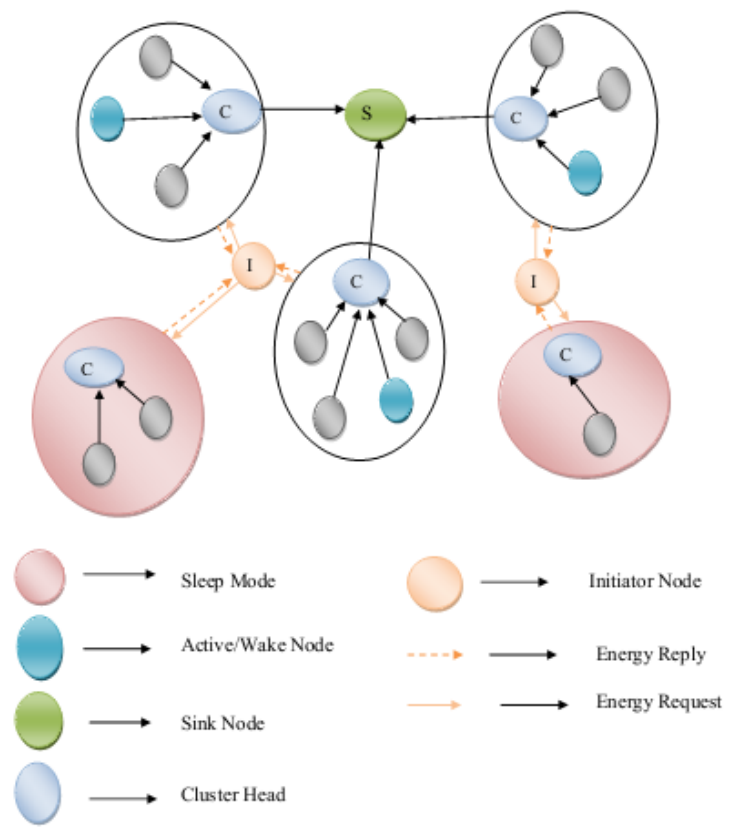

Figure 2: Sleep/Wake Scheduling

In the above Figure the initiator node in the network broadcasts an energy request message to all the clusters in the network in order to know the residual energy. Then the entire cluster sends an energy reply to the initiator node. By this way the initiator node collects the residual energy level of the clusters. The initiator node compares the clusters residual energy with the fixed standard threshold value, if any cluster's energy is less than the threshold value than that particular cluster will be sent to the sleep mode. For example consider in the above figure, let the threshold value be 10 and consider the cluster1's energy is level is 12, cluster2's is 13, cluster3's is 11 and let the cluster4's and the cluster5's be 5 and 6 . Now in this case after these values are collected by the initiator node it'll compare with the threshold. Because of this reason it can be seen that, only three clusters are transmitting data to the sink node and the $4^{\text {th }}$ and $5^{\text {th }}$ clusters are sent to the sleep mode.

\subsection{Algorithm}

Initially an initiator node randomly sends an energy request $\left(\mathrm{E}_{\mathrm{REQ}}\right)$ to all the surrounding nodes to collect the residual energy details of the nodes. The initiator node compares its energy with the node's energy. If the node's energy is more than the initiator node then the sensor node $\left(\mathrm{S}_{\mathrm{i}}\right)$ will send an energy reply $\left(\mathrm{E}_{\mathrm{REP}}\right)$ to the initiator node. Through this way the $\mathrm{CH}$ is decided and this $\mathrm{CH}$ broadcast the $\mathrm{CH}_{\mathrm{ADV}}$ to the sensor nodes. Each node sends a joint request $\left(\mathrm{J}_{\mathrm{REQ}}\right)$ to the $\mathrm{CH}$, accepting this request the $\mathrm{CH}$ sends a joint reply $\left(\mathrm{J}_{\mathrm{REP}}\right)$ to the sensor nodes and this way the cluster is formed. In a cluster the $\mathrm{CH}$ selects the node with high residual energy and keeps that node in active and sends the remaining nodes into the sleep mode. Then again the initiator node sends an $\mathrm{E}_{\mathrm{REQ}}$ to the cluster for their residual energy and this energy will be compared with the threshold value. If the energy value is less than the threshold value than the particular cluster will be sent to the sleep mode. And finally the transmission to the sink node will be done by the clusters in the network.

1. In a sensor network consider some nodes as initiator nodes to collect the residual energy details of the sensor nodes.
2. The initiator I1 selects the cluster head based upon the energy level information.

$$
\begin{aligned}
& \text { For each neighbor } \mathrm{Ni} \text { of } \mathrm{I} 1, \mathrm{i}=1,2 \ldots . \mathrm{r} \\
& \text { If } R L_{\mathrm{i}}>\mathrm{RL}_{\mathrm{ini}} \text {, then, } \\
& \mathrm{S}_{\mathrm{i}} \text { sends a reply message for energy }\left(\mathrm{E}_{\mathrm{REP}}\right) \text {. }
\end{aligned}
$$

Else

$\mathrm{S}_{\mathrm{i}}$ waits for cluster head advertisement messages $\left(\mathrm{CH}_{\mathrm{ADV}}\right)$. End For

3. I1 select the node $\mathrm{St}$ as $\mathrm{CH} 1$ such that $\mathrm{RLt}=\max \left\{R L_{\mathrm{i}}\right\}$, $\mathrm{i}=1,2 \ldots \mathrm{r}$

4. $\mathrm{CH} 1$ broadcast a $\mathrm{CH}_{\mathrm{ADV}}$ to $\mathrm{N}_{\mathrm{i}}$

5. Each node $\mathrm{N}_{\mathrm{i}}$ sends request $\mathrm{J}_{\mathrm{REQ}}$ to $\mathrm{CH} 1$.

6. On accepting $\mathrm{J}_{\mathrm{REP}}$ from the $\mathrm{CH}$, nodes $\mathrm{N}_{\mathrm{i}}$ join the cluster.

7. The cluster selects the node with high residual energy and keeps that node in active and sends the remaining nodes into the sleep mode.

8. The initiator node I1 send the energy request to clusters

If the RL (cluster) > threshold value

The cluster will be in active mode

Else

The cluster will be sent to the sleep mode

9. Transmit the data to the sink node

10. End

\section{SIMULATION RESULTS}

Cluster Based Sleep/Wakeup Scheduling Technique (CBSST) is evaluated through NS2 [16] simulation. A random network deployed in an area of 500 X $500 \mathrm{~m}$ is considered. Initially 100 sensor nodes are placed in square grid area by placing each sensor in a 50x50 grid cell. 10 cluster heads are deployed in the grid region according to our protocol. The sink is assumed to be situated 100 meters away from the above specified area. In the simulation, the channel capacity of mobile hosts is set to the same value: 2 Mbps. The simulated traffic is CBR with UDP source and sink. The number of sources is per cluster is varied fro 1 to 4 .

Table 1 summarizes the simulation parameters used

Table 1: Simulation Parameters

\begin{tabular}{|c|c|}
\hline No. of Nodes & 100 \\
\hline Area Size & 500 X 500 \\
\hline Mac & 802.11 \\
\hline Routing protocol & EEMD \\
\hline Simulation Time & $50 \mathrm{sec}$ \\
\hline Traffic Source & CBR \\
\hline Packet Size & 512 bytes \\
\hline Rate & 100 to $300 \mathrm{~kb}$ \\
\hline Transmission Range & $150 \mathrm{~m}$ \\
\hline No. of Clusters & 1 to 4 \\
\hline Transmit Power & $0.395 \mathrm{w}$ \\
\hline Receiving power & $0.660 \mathrm{w}$ \\
\hline Idle power & $0.035 \mathrm{w}$ \\
\hline Initial Energy & 7.1 Joules \\
\hline No. of clusters & 10 \\
\hline
\end{tabular}




\subsection{Performance Metrics}

The performance of CBSST technique is compared with EEMD technique [ ]. The performance is evaluated mainly, according to the following metrics.

- Average end-to-end delay: The end-to-end-delay is averaged over all surviving data packets from the sources to the destinations.

- Average Packet Delivery Ratio: It is the ratio of the number .of packets received successfully and the total number of packets transmitted.

- Energy: It is the average energy consumed for the data transmission.

- Drop: It is the number of packets dropped during the data transmission.

\subsection{Results}

\section{A. Based on Rate}

The transmission rate is varied as $100,150,200,250$ and $300 \mathrm{~Kb}$ for CBR traffic and the performance metrics are evaluated.

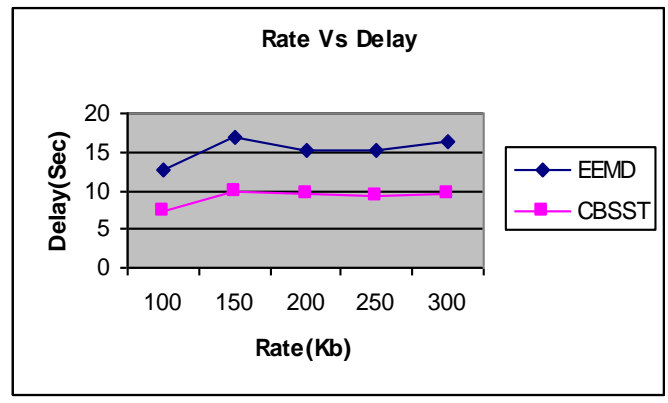

Figure 3: Rate Vs Delay

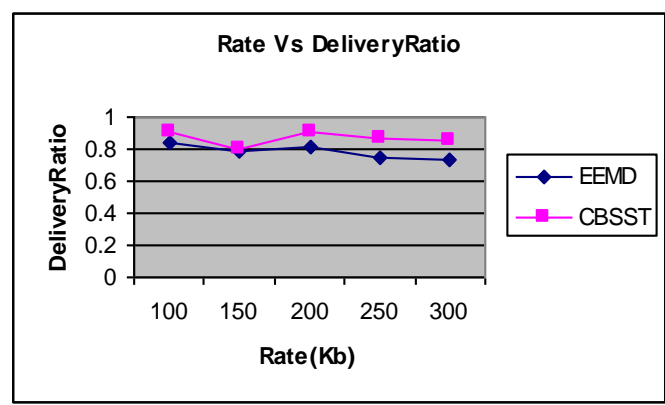

Figure 4: Rate Vs Delivery Ratio

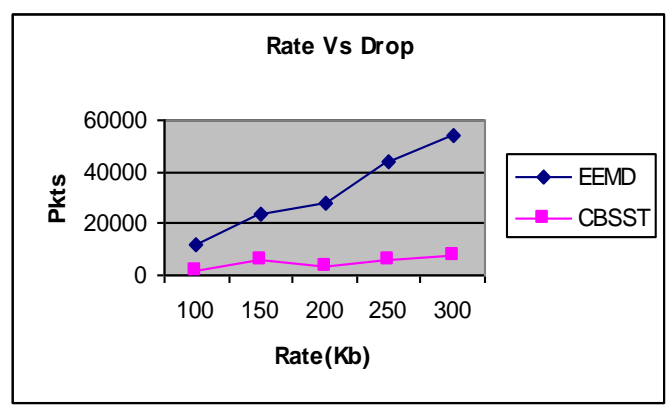

Figure 5: Rate Vs Drop

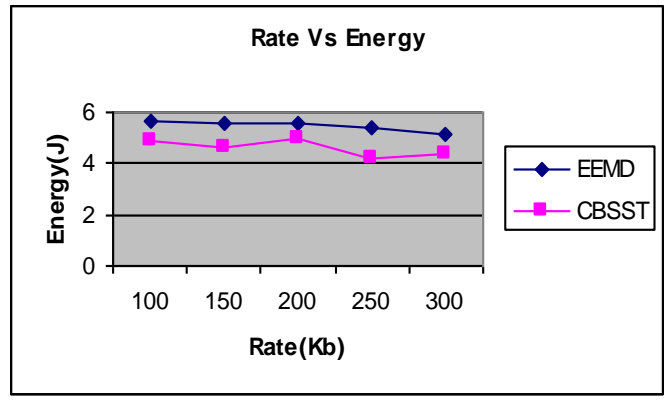

Figure 6: Rate Vs Energy

Figures 3-6 show the results for delay, packet delivery ratio, packet drop and energy consumption, respectively, for both EEMD and CBSST techniques, when the rate is increased. From the results, it can be seen that CBSST out performs EEMD.

\section{B. Based on Clusters}

The number of clusters is varied as 1,2,3 and 4 and the performance metrics are evaluated.

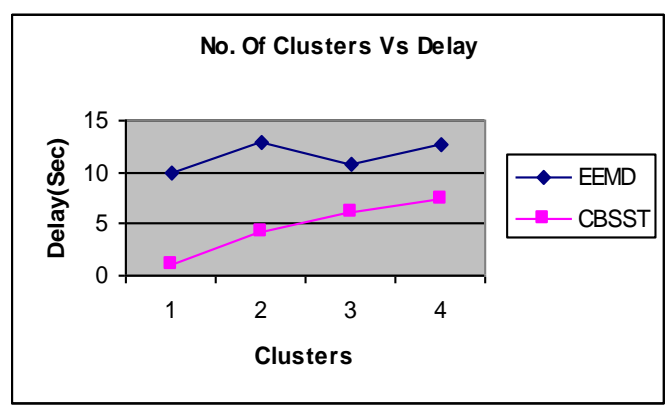

Figure 7: Clusters Vs Delay

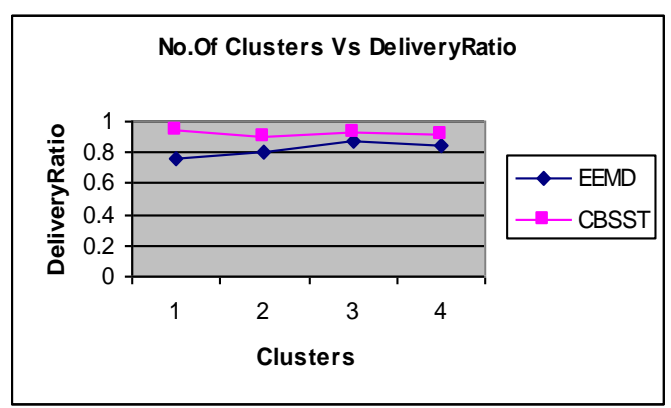

Figure 8: Clusters Vs Delivery Ratio 


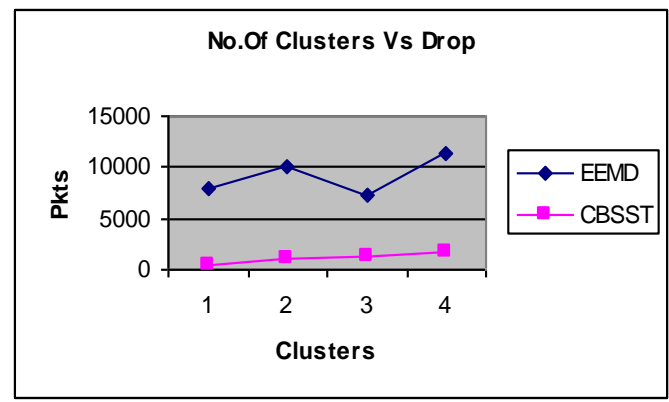

Figure 9: Clusters Vs Drop

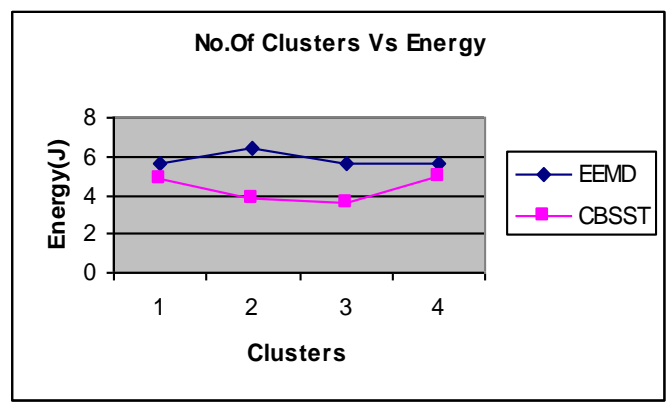

Figure 10: Clusters Vs Energy

Figures 7-10 show the results for delay, packet delivery ratio, packet drop and energy consumption, respectively, for both EEMD and CBSST techniques, when the number of clusters is increased. From the results, it can be seen that CBSST out performs EEMD.

\section{CONCLUSION}

In this paper, a cluster based Sleep/Wake scheduling technique is proposed. Initially, initiator nodes are considered for selecting the cluster head. The cluster head selects a node with the highest energy and keep it in active mode and sends the remaining nodes into the sleep mode. Then again the initiator nodes collect the residual energies details of these clusters and compare with the standard threshold value. If the cluster energy is less than the threshold value then that particular cluster will be sent into the sleep mode. By simulation results, it is proved that the proposed technique reduces the energy consumption and increases the packet delivery ratio.

\section{REFERENCES}

[1] Amir Sepasi Zahmati, Bahman Abolhassani, Ali Asghar Beheshti Shirazi, and Ali Shojaee Bakhtiari, "An EnergyEfficient Protocol with Static Clustering for Wireless Sensor Networks", International Journal of Electronics, Circuits and Systems Volume 1 Number 2, 2008

[2] Olfa Gaddour, Anis Koub^aa and Mohamed Abid, "SeGCom: A Secure Group Communication Mechanism in Cluster-Tree Wireless Sensor Networks", Communications and Networking, ComNet, 3-6 Nov. 2009

[3] T.Kavitha, D.Sridharan, "Security Vulnerabilities in Wireless Sensor Networks: A Survey", Journal of Information Assurance and Security, vol 5, p-031-044, 2010

[4] Guofang Nan, Guanxiong Shi, Zhifei Mao and Minqiang $\mathrm{Li}$ "CDSWS: coverage-guaranteed distributed sleep/ wake scheduling for wireless sensor networks", EURASIP
Journal on Wireless Communications and Networking 2012

[5] Francesco De Pellegrini, Karina Gomez, Daniele Miorandi and Imrich Chlamtac "Distributed Wake-Up Scheduling for Energy Saving in Wireless Networks", 2011

[6] Sha Liu, Kai-Wei Fan, Prasun Sinha "Dynamic Sleep Scheduling using Online Experimentation for Wireless Sensor Networks", 2005

[7] Yan Wu, Sonia Fahmy, Ness B. Shroff "Sleep/Wake Scheduling for Multi-hop Sensor Networks: Nonconvexity and Approximation Algorithm", October 7 , 2009

[8] Chul-Ho Lee and Do Young Eun "A Distributed Wake-up Scheduling for Opportunistic Forwarding in Wireless Sensor Networks", 2010

[9] Yan Wu, Sonia Fahmy, Ness B. Shroff "Optimal Sleep/Wake Scheduling for Time-Synchronized Sensor Networks with QoS", 2006

[10] Bo Jiang, Binoy Ravindran and Hyeonjoong Cho "Energy Efficient Sleep Scheduling in Sensor Networks for Multiple Target Tracking”, 2009

[11] Chih-Min Chao1 and Yi-Wei Lee, "A Quorum-Based Energy Saving MAC Protocol Design for Wireless Sensor Networks", IEEE, 2009

[12] Nagamalar, T. and T.R. Rangaswamy, "Sleeping Cluster based Medium Access Control Layer Routing Protocol for Wireless Sensor Networks", Journal of Computer Science 8 (8): 1294-1303, 2012

[13] Sumit Kumar and Siddhartha Chauhan, "A Survey on Scheduling Algorithms for Wireless Sensor Networks", International Journal of Computer Applications (0975 8887) Volume 20- No.5, April 2011

[14] Siddhartha Chauhan and Lalit Kumar Awasthi, "Cluster Based Task Scheduling in Wireless Sensor Network", International Journal of Computer Applications (0975 8887) Volume 33- No.4, November 2011

[15] S.V. Manisekaran and R. Venkatesan, "Power Efficient Scheduling Technique for Multiple Sinks in Wireless Sensor Networks", European Journal of Scientific Research, Vol. 89 No 2, pp.191-202, October, 2012.

[16] Sasikala V, and C. Chandrasekar, "Energy Efficient Multipath Data Fusion Technique for Wireless Sensor Networks", ACEEE Int. J. on Network Security, Vol. 03, No. 02, April 201

[17] Network Simulator: http://www.isi.edu/nsnam/ns

Sasikala $\mathbf{V}$ received the MCA., degree from University of Madras, Chennai, Tamilnadu, the M.Phil., degree in Computer Science from Manonmaniam Sundaranar University, Tirunelveli, Tamilnadu. She is pursuing her $\mathrm{PhD}$ under the guidance of Dr. C. Chandrasekar and currently working as Assistant Professor \& HOD of Computer Application Department in DRBCCC Hindu College, Chennai, Tamil Nadu, India.

Dr. C. Chandrasekar received his Ph.D. degree from Periyar University, Salem, TN, India. He has been working as Associate Professor at Dept. of Computer Science, Periyar University, Salem - 636 011, Tamil Nadu, India. The topic of his doctoral dissertation was "An Optimization and Seamless Mobility for Integration of Wireless LAN and Cellular Networks". His research interest includes Wireless networking, Mobile computing, Computer Communication and Networks. He was a Research guide at various universities in India. $\mathrm{He}$ has been published more than 50 research papers at various National / International Journals. 